\title{
Prognostic value of estimated glomerular filtration rate in hospitalised older patients (over 65) with COVID-19: a multicentre, European, observational cohort study
}

\author{
Ben Carter ${ }^{1 \dagger}$, Euan A. Ramsay ${ }^{2 \dagger}$, Roxanna Short ${ }^{3}$, Sarah Goodison ${ }^{4}$, Jane Lumsden ${ }^{5}$, Amarah Khan ${ }^{6}$, \\ Philip Braude ${ }^{7}$, Arturo Vilches-Moraga ${ }^{6}$, Terence J. Quinn ${ }^{8}$, Kathryn McCarthy ${ }^{7}$, Jonathan Hewitt ${ }^{9^{*}+} \mathbb{C}$, \\ Phyo K. Myint ${ }^{2+}$ and On Behalf of COPE Study
}

\begin{abstract}
Background: The reduced renal function has prognostic significance in COVID-19 and it has been linked to mortality in the general population. Reduced renal function is prevalent in older age and thus we set out to better understand its effect on mortality.

Methods: Patient clinical and demographic data was taken from the COVID-19 in Older People (COPE) study during two periods (February-June 2020 and October 2020-March 2021, respectively). Kidney function on admission was measured using estimated glomerular filtration rate (eGFR). The primary outcomes were time to mortality and 28-day mortality. Secondary outcome was length of hospital stay. Data were analysed with multilevel Cox proportional hazards regression, and multilevel logistic regression and adjusted for individual patient clinical and demographic characteristics.

Results: One thousand eight hundred two patients (55.0\% male; median [IQR] 80 [73-86] years) were included in the study. 28 -day mortality was $42.3 \%(n=742) .48 \%(n=801)$ had evidence of renal impairment on admission. Using a time-to-event analysis, reduced renal function was associated with increased in-hospital mortality (compared to eGFR $\geq 60$ [Stage 1\&2]): eGFR 45-59 [Stage 3a] aHR=1.26 (95\%Cl 1.02-1.55); eGFR 30-44 [Stage 3b] aHR=1.41 (95\%Cl 1.14-1.73); eGFR 1-29 [Stage 4\&5] aHR $=1.42$ (95\%Cl 1.13-1.80). In the co-primary outcome of 28-day mortality, mortality was associated with: Stage 3a adjusted odds ratio $(\mathrm{aOR})=1.18(95 \% \mathrm{Cl} 0.88-1.58)$, Stage $3 \mathrm{~b}$ aOR $=1.40(95 \% \mathrm{Cl}$ 1.03-1.89); and Stage $4 \& 5 \mathrm{aOR}=1.65(95 \% \mathrm{Cl} 1.16-2.35)$.
\end{abstract}

Conclusion: eGFR on admission is a good independent predictor of mortality in hospitalised older patients with COVID-19 population. We found evidence of a dose-response between reduced renal function and increased mortality.

Keywords: COVID-19, Chronic kidney failure, eGFR, Mortality, Dose-response

*Correspondence: HewittJ2@cardiff.ac.uk

†Ben Carter, Euan A. Ramsay, Jonathan Hewitt and Phyo K. Myint contributed equally to this work.

${ }^{9}$ Division of Population Medicine, Cardiff University, Cardiff Cf10 3XQ, UK

Full list of author information is available at the end of the article

\section{Introduction}

Initially, COVID-19 was described as primarily respiratory in nature and involvement of kidneys was not widely reported [1, 2]. However, further literature has described the increased presence of worsening kidney function original author(s) and the source, provide a link to the Creative Commons licence, and indicate if changes were made. The images or other third party material in this article are included in the article's Creative Commons licence, unless indicated otherwise in a credit line to the material. If material is not included in the article's Creative Commons licence and your intended use is not permitted by statutory regulation or exceeds the permitted use, you will need to obtain permission directly from the copyright holder. To view a copy of this licence, visit http://creativecommons.org/licenses/by/4.0/. The Creative Commons Public Domain Dedication waiver (http://creativeco mmons.org/publicdomain/zero/1.0/) applies to the data made available in this article, unless otherwise stated in a credit line to the data. 
concurrent with COVID-19 infection [3]. A number of potential mechanisms of kidney injury have been described, including direct viral infection of the kidneys, leading to acute tubular injury and endothelial damage as well as mechanisms secondary to systemic illness including sepsis and hypovolaemia $[4,5]$.

Estimated glomerular filtration rate (eGFR), is a calculation based on serum creatinine, age, race, sex and body size, and is used clinically as a measure of kidney function [6]. It is well described as a good indicator of mortality in non-COVID-19 patients with both acute and chronic kidney disease (CKD) [7] and in COVID19 patients [8]. Lower baseline eGFR has also been shown to lead to increased rates of acute kidney injury (AKI), and renal replacement therapy in COVID-19 patients [9]. Lower eGFR was also more commonly seen in multimorbid patients and older people [10]. Several studies have reported a decline in renal function as a binary threshold of eGFR is associated with increased mortality in COVID-19 patients [10, 11] but few have reported on the association between the increasing severity of categorised eGFR and mortality in COVID-19 [12, 13].

Previous studies in older adults ( $>65$ years) showed that prevalence of CKD on admission with COVID-19 was $11.4 \%$ [14], and development of AKI ranged between $24.8 \%$ [14] and 39\% [14]. Indeed, older age and eGFR (less than 60) [12] have been well described as a risk factors for mortality [15-17] alongside AKI development [18] and subsequent mortality in COVID-19 [19]. In addition, older age is associated with increased serum creatinine levels on admission in COVID-19 patients [20]. To date only the Geriatric Medicine Research Collaborative [13] and Xu et al. [21] have explored the gradated relationship between eGFR decline and mortality in older adults with COVID-19.

The aim of this paper is to determine the relationship between eGFR on admission to hospital with COVID19 infection and clinical outcomes including mortality, and length of stay in older adults, using data from the COVID-19 in Older People (COPE) Study [16].

\section{Methods}

\section{Study population and setup}

This study was an extension of the COVID-19 in Older People (COPE) Study, with additional participants included in the second wave. The primary COPE study was a multicentre, observational study with 13 centres in both the UK and Italy [16]. The study protocol for the original COPE study has previously been published [22], with this study following the same study design. Approval for the study was granted in the UK by the Health
Research Authority (20/HRA/1898) and in Italy from the Ethics Committee of Hospital Policlinico Modena (Reference 369/2020/OSS/AOUMO). Data was collected using a standardised case report based on hospital records and entered into a centrally co-ordinated InferMed MACRO database housed within King's College London. Data protection policies were adhered to at each hospital.

\section{Participants}

Consecutive patients aged 65 years or older, who were admitted to hospital at any of the recruiting centres during the first wave (27th February to 10th June 2020) and the second wave (1st October 2020 - 8th March 2021) with a COVID-19 diagnosis were included in the present study. Patients aged 65 years or older who developed COVID-19 whilst already hospitalised for a different reason (nosocomial infections) were also included. Nosocomial infection was assumed if the date of diagnosis was more than 5 days after the date of admission [23]. Diagnostic criteria for COVID-19 included a laboratory confirmed positive swab for SARS-CoV-2, and a clinical diagnosis based on signs, symptoms and radiological reporting consistent with COVID-19. There were no exclusion criteria applied.

\section{Outcomes}

The primary outcome was mortality (time-to-mortality and 28-day mortality). The secondary outcome was length of stay in hospital (time from admission, or from diagnosis for nosocomial cases to discharge). Patients who were discharged prior to day- 28 were imputed as having survived at day 28 . Patients who died were censored in the time-to-discharge analysis.

\section{Primary exposure}

Renal function was the primary exposure under investigation assessed as eGFR (CKD-EPI) on admission and was categorised into: Stage 1\&2 (normal kidney function to mild loss of kidney function) (eGFR $\geq 60 \mathrm{ml} /$ $\mathrm{min} / 1.73 \mathrm{~m}^{2}$ ); Stage 3a (mild to moderate loss of kidney function, eGFR $\left.45-59 \mathrm{ml} / \mathrm{min} / 1.73 \mathrm{~m}^{2}\right)$; Stage $3 \mathrm{~b}(\mathrm{mod}-$ erate to severe loss of kidney function, eGFR $30-44 \mathrm{ml} /$ $\mathrm{min} / 1.73 \mathrm{~m}^{2}$ ); Stages 4 and 5 (Severe to complete loss of kidney function, eGFR $1-29 \mathrm{ml} / \mathrm{min} / 1.73 \mathrm{~m}^{2}$ ) [24].

\section{Covariates}

Clinical characteristics collected included: sex; age, smoking status (current smoker, previous smoker and never smoker); C-reactive protein (CRP) levels at admission [25] and a diagnosis of diabetes mellitus, hypertension or coronary artery disease (CAD) present at admission. Patient's frailty was assessed in-hospital, 
based on frailty status 2 weeks prior to admission using the Clinical Frailty Scale (CFS) [26, 27].

\section{Terminally ill patients}

Due to very few patients with a terminal illness (CFS 9) being included in the study, they were excluded from analyses.

\section{Statistical analysis}

There were 55 patients with missing smoking data, who were recorded as 'never smokers', and a further 64 patients with a missing eGFR recording, inputted as having an eGFR $\geq 60$. Clinical characteristics from both waves were compared by in hospital mortality. Time-toevent outcomes (mortality and time to discharge) were analysed with multilevel multivariable Cox proportional hazards $(\mathrm{PH})$ regression models. Each Cox regression model fitted the hospital site as a random intercept effect, to account for heterogeneity across sites. Crude hazard ratios (HR) and adjusted hazard ratios (aHR) are presented alongside the associated 95\% Confidence Intervals $(\mathrm{CI})$. The $\mathrm{PH}$ assumption was assessed visually using $\log -\log$ plots. Analyses were performed using Stata SE version 16 (StataCorp LLC; College Station, TX), KaplanMeier and subgroup forest plots were visualised in R.

28-day mortality was analysed with a multivariable multilevel logistic regression model, which fitted hospital site as a random effect, with crude Odds Ratios (OR) and adjusted Odds Ratios (aOR) and associated 95\% CIs. All models were adjusted for: eGFR; wave 1 or wave 2; age (categorised into: 65-74years, 75-84years, 85-94 years, $\geq 95$ years); sex (male or female); smoking status (never smoker, current smoker or previous smoker); diabetes (yes or no); hypertension (yes, yes and on treatment or no); coronary artery disease (yes or no); C-reactive protein on admission $(0-39 \mathrm{mg} / \mathrm{dl}$ or $\geq 40 \mathrm{mg} / \mathrm{dL}$ [25]); Clinical Frailty Scale (categorised into: CFS 1-3, CFS 4, CFS 5-6, CFS 7-8, CFS 9).

\section{Dose-response}

This was assessed in each analysis using a test for post estimation linear test for trend to the adjusted analyses and presented as the linear change from each category of renal failure compared to Stage1\&2.

\section{Subgroup analyses}

Subgroups analyses were carried out for each outcome using the multivariable multilevel analyses as above. These present associations of eGFR (categorised using the established binary cut off for reduced renal function: eGFR $1-59 \mathrm{ml} / \mathrm{min} / 1.73 \mathrm{~m}^{2}$, eGFR $\geq 60 \mathrm{ml} / \mathrm{min} / 1.73 \mathrm{~m}^{2}$ ) with mortality and time-to-discharge, within each subgroup.

\section{Results}

A total of 1802 patients aged $\geq 65$ years were included in the study (Wave 1, $n=1318$; Wave $2, n=484$ ). The mean age was 79.6 (range 65-101, SD 7.98), and 992 (55.0\%) were male (Table 1). The median (IQR) time between admission and mortality was 14 days [7-27]. All cause in-hospital 28 -day mortality was $42.3 \%(n=742)$. 28-day mortality was higher in older age groups $(56.5 \%$ in those aged $95+$ years; $48.2 \%$ in those aged $85-94 ; 42.8 \%$ in those aged $75-84 ; 31.2 \%$ in those aged $65-74$ ), in patients at increasing stages of renal failure on admission $(53.5 \%$ at Stages 4 and 5; $48.3 \%$ at Stage 3b; $42.7 \%$ at Stage 3A; $36.0 \%$ at Stages 1 and 2, Fig. 1), and patients with comorbidities including coronary artery disease $(45.6 \%$ vs $39.6 \%$ ) and diabetes (42.6\% vs $40.6 \%$ ), and in those with an increased frailty score (Table 1 ).

\section{Primary outcomes: time to mortality and 28-day mortality} In the crude Cox proportional hazards regression, eGFR was associated with increased mortality (Table 2). In the multilevel multivariable Cox $\mathrm{PH}$ regression, reduced renal function was associated with increased mortality (compared to Stages 1 and 2): Stage $3 \mathrm{a}$ aHR $=1.26$ $(95 \%$ CI $1.02-1.55)$; Stage $3 \mathrm{~b}$ aHR $=1.41(95 \%$ CI $1.14-$ 1.73); Stages 4 and 5 aHR $=1.42(95 \%$ CI $1.13-1.80)$ (Table 2, Fig. 1). In addition to this increasing age, male sex, CRP $\geq 40 \mathrm{mg} / \mathrm{dL}$, and a CFS score $\geq 5$ were associated with increased mortality (Table 2). There was very clear evidence of a linear test for trend $(\mathrm{aHR}=-0.13$; $95 \%$ CI $-0.21,-0.05 ; p=0.002$ ).

For 28-day mortality, similar findings were reported with a clearer dose-response for worsening renal function linked to increased mortality (Table 3 ). The Stage $3 \mathrm{a}$ adjusted odds ratio $(\mathrm{aOR})=1.18(95 \% \mathrm{CI} 0.88-1.58)$; Stage $3 \mathrm{~b}$ aOR $=1.40$ (95\%CI 1.03-1.89); Stages 4 and $5 \mathrm{aOR}=1.65(95 \% \mathrm{CI} 1.16-2.35)$. From the covariates increasing age, male sex, CRP $>40 \mathrm{mg} / \mathrm{dl}$, and increasing frailty were associated with increased mortality in a multilevel logistic regression (Table 3). There was very clear evidence of a linear test for trend $(\mathrm{aOR}=-0.19 ; 95 \% \mathrm{CI}$ $-0.31,-0.06 ; p=0.003$ ).

\section{Secondary outcome: time to discharge}

There was no association, in either the crude or adjusted analysis, between stage of kidney disease and time to 
Table 1 Included Population description

\begin{tabular}{|c|c|c|c|}
\hline & $\begin{array}{l}\text { Alive } \\
(N=1060)\end{array}$ & $\begin{array}{l}\text { Dead } \\
(N=742)\end{array}$ & $\begin{array}{l}\text { Total } \\
(N=1802)\end{array}$ \\
\hline & $\mathrm{N}(\%)$ & N (\%) & N (\%) \\
\hline \multicolumn{4}{|l|}{ eGFR } \\
\hline $1-29$ & $92(46.5)$ & $106(53.5)$ & $198(11.0)$ \\
\hline $30-44$ & $153(51.7)$ & $143(48.3)$ & $296(16.4)$ \\
\hline $45-59$ & $176(57.3)$ & $131(42.7)$ & $307(17.0)$ \\
\hline $60+$ & $600(64.0)$ & $337(36.0)$ & $937(52.0)$ \\
\hline Missing & 39 & 25 & 64 \\
\hline \multicolumn{4}{|l|}{ Wave } \\
\hline 1 & 765 (58.0) & $553(42.0)$ & $1318(73.1)$ \\
\hline 2 & $295(61.0)$ & $189(39.0)$ & $484(26.9)$ \\
\hline \multicolumn{4}{|l|}{ Age } \\
\hline $65-74$ & $362(68.8)$ & 164 (31.2) & $526(29.2)$ \\
\hline $75-84$ & $431(57.2)$ & $322(42.8)$ & $753(41.8)$ \\
\hline $85-94$ & $247(51.8)$ & $230(48.2)$ & $477(26.5)$ \\
\hline $95+$ & $20(43.5)$ & $26(56.5)$ & $46(2.6)$ \\
\hline \multicolumn{4}{|l|}{ Sex } \\
\hline Female & 509 (62.9) & $300(37.1)$ & 809 (44.9) \\
\hline Male & $550(55.4)$ & $442(44.6)$ & $992(55.0)$ \\
\hline Missing & 1 & 0 & 1 \\
\hline \multicolumn{4}{|l|}{ Smoking } \\
\hline Never Smokers & $515(60.8)$ & $332(39.2)$ & $847(47.0)$ \\
\hline Ex-smokers & $444(55.7)$ & $353(44.3)$ & $797(44.2)$ \\
\hline Current Smokers & $67(65.0)$ & $36(35.0)$ & $103(5.7)$ \\
\hline Missing & 34 & 21 & 55 \\
\hline \multicolumn{4}{|l|}{ Diabetes } \\
\hline No & $764(59.4)$ & $522(40.6)$ & $1286(71.4)$ \\
\hline Yes & $294(57.4)$ & $218(42.6)$ & $512(28.4)$ \\
\hline Missing & 2 & 2 & 4 \\
\hline \multicolumn{4}{|l|}{ Hypertension } \\
\hline No & $452(57.4)$ & $335(42.6)$ & $787(43.7)$ \\
\hline Yes & $181(61.1)$ & 115 (38.9) & $296(16.4)$ \\
\hline Yes \& on treatment & $427(59.4)$ & $292(40.6)$ & 719 (39.9) \\
\hline \multicolumn{4}{|l|}{ CAD } \\
\hline No & $807(60.4)$ & $528(39.6)$ & $1335(74.1)$ \\
\hline Yes & $252(54.4)$ & $211(45.6)$ & $463(25.7)$ \\
\hline Missing & 1 & 3 & 4 \\
\hline \multicolumn{4}{|l|}{ CRP } \\
\hline $0-40$ & $401(69.4)$ & $177(30.6)$ & $578(32.1)$ \\
\hline$>40$ & $659(53.8)$ & $565(46.2)$ & $1224(67.9)$ \\
\hline \multicolumn{4}{|l|}{ CFS } \\
\hline CFS 1-3 & $283(71.1)$ & $115(28.9)$ & $398(22.1)$ \\
\hline CFS 4 & $157(59.5)$ & $107(40.5)$ & $264(14.7)$ \\
\hline CFS 5-6 & $368(57.4)$ & $273(42.6)$ & $641(35.6)$ \\
\hline CFS 7-8 & $227(50.9)$ & $219(49.1)$ & $446(24.8)$ \\
\hline CFS 9 & $9(29.0)$ & $22(71.0)$ & $31(1.7)$ \\
\hline Missing & 16 & 6 & 22 \\
\hline
\end{tabular}

discharge. Compared to Stages 1 and 2, the adjusted analysis found a relationship between renal function and time to discharge: Stage $3 \mathrm{a}$ aHR $=1.09$ (95\%CI 0.89-1.32); Stage $3 \mathrm{~b}$ aHR $=1.09$ (95\%CI 0.88-1.35); Stages 4 and 5 $\mathrm{aHR}=0.84$ (95\%CI 0.64-1.09) (Table 4). There was no evidence for any dose-response $(p=0.21)$.

\section{Subgroup analysis}

Subgroup analyses of individuals with Stage 3a-5 kidney disease that were: first wave patients; aged 65-74 and 85-94; female sex; never and current smoker; CRP $\geq 40 \mathrm{mg} / \mathrm{dL}$; no hypertension; no diabetes; CFS 4 and CFS 5-6 were associated with increased mortality (Additional file 1: Fig. 1). 28-day mortality subgroup analysis of those with a Stage 3a-5 kidney disease found that: first wave patients; aged 85-94, female sex; never and current smoker; CRP $\geq 40 \mathrm{mg} / \mathrm{dL}$; no hypertension; no diabetes; coronary artery disease; and CFS 4 were associated with increased mortality (Additional file 1: Fig. 2). On subgroup analysis of those with Stage 3a-5 kidney disease, no patient characteristics were associated with length of stay in hospital (Additional file 1: Fig. 3).

\section{Discussion}

Our study included 1802 older patients during waves one and two in Europe and we found $41.1 \%$ of them died by day 28 . Within both the time-to-mortality and 28-day analysis we found a suspected dose-response effect of eGFR, between CKD Stages $1 \& 2$ and 3b, 4 and 5 with increasing effect, and this appears to be the first study to report this finding within this cohort. Whilst the majority of previous studies have shown the relationship between eGFR and mortality using a binary comparison comparing Stages 1 \& 2 versus Stages 3a, 3b, 4, and 5 combined [12, 28], and few have assessed the association comparing groups $3 \mathrm{a}, 3 \mathrm{~b}, 4$, and 5 separately allowing us to assess the likely dose response. Previous work by the Geriatric Medicine Research Collaborative [13] included 5711 individuals and investigated the effect of eGFR categorised in each stage and only found an association in Stages 4 and 5. Our findings extend this work as we report an association between mortality and Stages $3 \mathrm{~b}, 4$ and 5 . The recent international multicentre HOPE study (Health Outcome Predictive Evaluation for COVID 19) [12] looked at patients of all ages (mean age 66 years old) [12]. They reported that only $8.5 \%$ of patients had documented CKD before admission whereas 35\% had evidence of renal dysfunction on admission. The study similarly concluded that estimated renal function on admission, documented as eGFR, acted as an independent prognostic factor for mortality in a suspected doseresponse pattern [12]. 


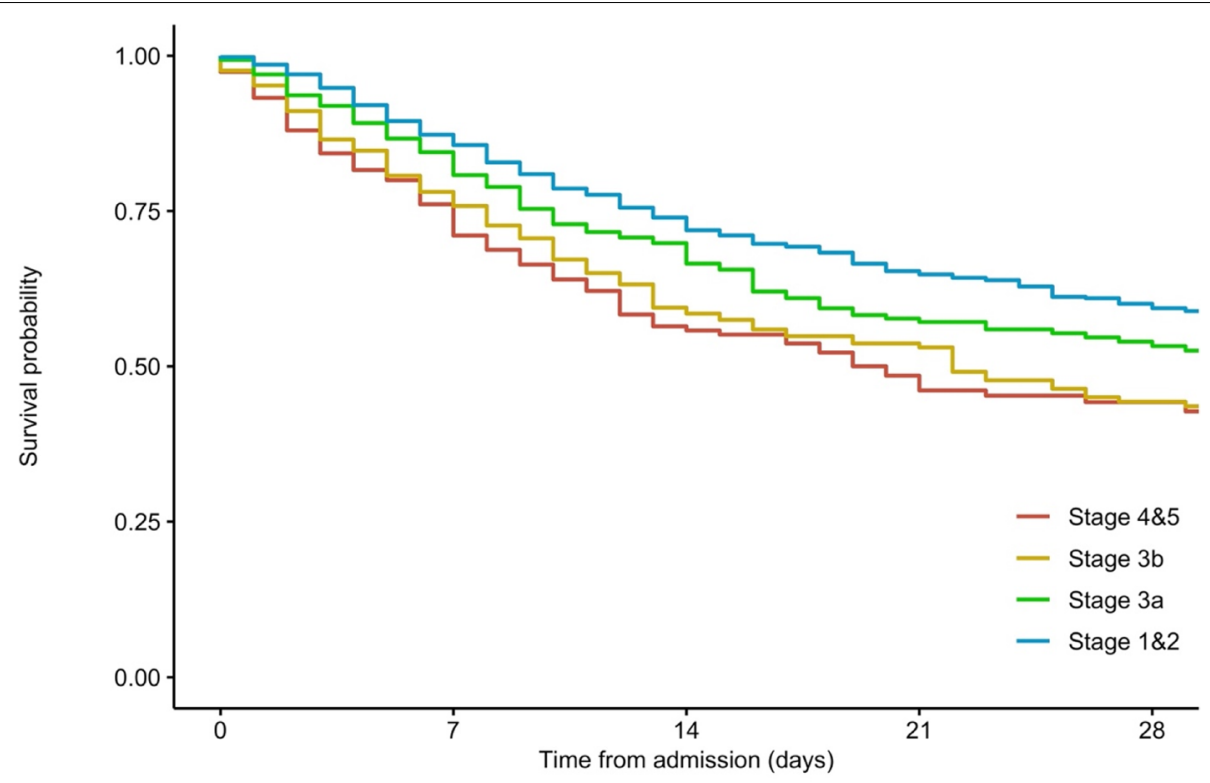

\begin{tabular}{|c|c|c|c|c|c|}
\hline \multicolumn{6}{|c|}{ Number at risk (number censored) } \\
\hline Stage $4 \& 5$ & $192(0)$ & $136(14)$ & $85(29)$ & $61(43)$ & $31(68)$ \\
\hline Stage $3 b$ & $294(2)$ & $207(33)$ & $123(69)$ & $85(95)$ & $60(104)$ \\
\hline Stage $3 a$ & $303(3)$ & $229(37)$ & $148(77)$ & $102(98)$ & $75(116)$ \\
\hline Stage $1 \& 2$ & $982(8)$ & $782(106)$ & 545 (232) & 372 (339) & $256(420)$ \\
\hline & 0 & 7 & 14 & 21 & 28 \\
\hline
\end{tabular}

Fig. 1 A Kaplan Meier survival function to assess categorised admission eGFR \& on the time to mortality for patients hospitalised with COVID-19

Whilst we identified a dose-response relationship for time to mortality outcome, the greatest association was with an eGFR $<45$ (Stages 3b, 4 and 5). This is in line with NICE guidance [29], that states an eGFR of $<45$ is an additional risk factor for development of AKI in COVID19 patients. The results showed that renal function was as strong a predictor of mortality as other key risk factors, such as frailty and age. Therefore, eGFR at $<45$ on admission needs to be considered with clinical relevance where identified.

Additionally, we found no association between renal failure and length of stay which was consistent with those from Geriatric Medicine Research Collaborative [13] as neither study report an effect, possibly due to confounding from early mortality.

A number of potential mechanisms of kidney damage in COVID-19 have been hypothesised, including direct viral infection of the kidney via expression of ACE2 receptors in renal cells allowing virus entry which could lead to acute tubular injury and endothelial damage [30]. Damage secondary to cytokine mediated hyperinflammation and thrombotic microangiopathy [31, 32] and systemic illness including sepsis and hypovolaemia $[4,5]$ has also been described. Age and hypertension specifically, have been associated with increased renal dysfunction and susceptibility to AKI in Covid-19 [11]. Chronic renal impairment is also associated with increased RAAS activity and ACE2 receptors which could also predispose to easier Covid-19 direct cell infection in the kidney.

Our findings should be interpreted in the light of a number of limitations. First, we did not account for underlying renal disease in the patient cohort, therefore renal function calculated on admission did not stipulate between an eGFR due to acute deterioration or chronic renal impairment. Second, this study used a single measurement of kidney function, and did not collect data on longer term kidney function. We also were not able to account for the varying permutations of medications that patients were exposed to. However, so far, this is the largest study to explore the effect of renal impairment in older adults across waves 1 and 2 of the Pandemic, reporting a biologically plausible dose-response.

Our study findings offer important clinical implications, since COVID-19 is anticipated to be embedded as an endemic disease, with new variants circulating 
Table 2 Crude and multivariable Cox proportional hazards regression, presenting the crude Hazard Ratio (HR) and adjusted $H R(a H R)$ for the time to mortality

\begin{tabular}{|c|c|c|c|c|}
\hline & \multicolumn{2}{|l|}{ Crude } & \multicolumn{2}{|l|}{ Multivariable } \\
\hline & $\mathrm{HR}(95 \% \mathrm{Cl})$ & $p$ & aHR & $p$ \\
\hline \multicolumn{5}{|l|}{ eGFR (60+) } \\
\hline $1-29$ & $1.55(1.24-1.95)$ & $<0.001$ & $1.42(1.13-1.80)$ & 0.0031 \\
\hline $30-44$ & $1.54(1.26-1.89)$ & $<0.001$ & $1.41(1.14-1.73)$ & 0.0014 \\
\hline $45-59$ & $1.30(1.06-1.60)$ & 0.0125 & $1.26(1.02-1.55)$ & 0.0295 \\
\hline Wave 2 & $0.75(0.62-0.91)$ & 0.0036 & $0.77(0.63-0.94)$ & 0.0093 \\
\hline \multicolumn{5}{|l|}{ Age (65-74) } \\
\hline $75-84$ & $1.52(1.25-1.85)$ & $<0.001$ & $1.44(1.17-1.77)$ & $<0.001$ \\
\hline $85-94$ & $1.78(1.44-2.21)$ & $<0.001$ & $1.60(1.27-2.01)$ & $<0.001$ \\
\hline $95+$ & $2.74(1.78-4.22)$ & $<0.001$ & $2.61(1.67-4.09)$ & $<0.001$ \\
\hline Male & $1.13(0.97-1.32)$ & 0.1203 & $1.20(1.02-1.41)$ & 0.0279 \\
\hline \multicolumn{5}{|l|}{ Smoking (Never) } \\
\hline Ex-smoker & $1.22(1.05-1.42)$ & 0.0112 & $1.14(0.97-1.33)$ & 0.1204 \\
\hline Current smoker & $0.96(0.67-1.38)$ & 0.8157 & $0.94(0.65-1.37)$ & 0.7450 \\
\hline $\mathrm{CRP}>40$ & $1.81(1.52-2.16)$ & $<0.001$ & $1.81(1.51-2.16)$ & $<0.001$ \\
\hline Diabetes & $1.03(0.88-1.22)$ & 0.6902 & $0.99(0.83-1.17)$ & 0.8650 \\
\hline$C A D$ & $1.17(0.99-1.38)$ & 0.0722 & $1.03(0.87-1.23)$ & 0.7170 \\
\hline \multicolumn{5}{|l|}{ Hypertension (No) } \\
\hline Yes & $0.90(0.72-1.12)$ & 0.3506 & $0.91(0.72-1.14)$ & 0.4008 \\
\hline $\begin{array}{l}\text { Yes \& on treat- } \\
\text { ment }\end{array}$ & $0.86(0.72-1.01)$ & 0.0682 & $0.85(0.71-1.01)$ & 0.0653 \\
\hline \multicolumn{5}{|l|}{ CFS (1-3) } \\
\hline CFS 4 & $1.39(1.06-1.81)$ & 0.0158 & $1.28(0.97-1.67)$ & 0.0791 \\
\hline CFS 5-6 & $1.48(1.18-1.86)$ & $<0.001$ & $1.30(1.02-1.66)$ & 0.0327 \\
\hline CFS 7-8 & $1.91(1.50-2.43)$ & $<0.001$ & $1.63(1.26-2.11)$ & $<0.001$ \\
\hline
\end{tabular}

Note: aHR adjusted for eGFR, wave, age, sex, smoking status, CRP, diabetes, CAD, hypertension and CFS

globally [33]. Older adults are generally susceptible to COVID-19 and our results improve the identification of older patients with COVID-19 at risk of deterioration, to allow earlier review of risk factors and interventions aimed at preserving and correcting renal dysfunction where possible. Early recognition of renal impairment in older people should inform assessments of prognosis and, where appropriate, inform care escalation decisions. The clear association with deterioration of renal function and increasing age, represents both physiological changes, and also the effect of increased incidence of comorbidity; particularly hypertension, vascular disease and diabetes [34]. In addition, the presence of chronic renal impairment can lead to increased susceptibility to infection [35]. It should be highlighted that older patients may not be suitable for more invasive medical management, including critical care and renal replacement therapy. Therefore, it is even more
Table 3 Multilevel logistic regression, presenting the crude Odds Ratio (OR) and adjusted OR (aOR) for 28-day mortality

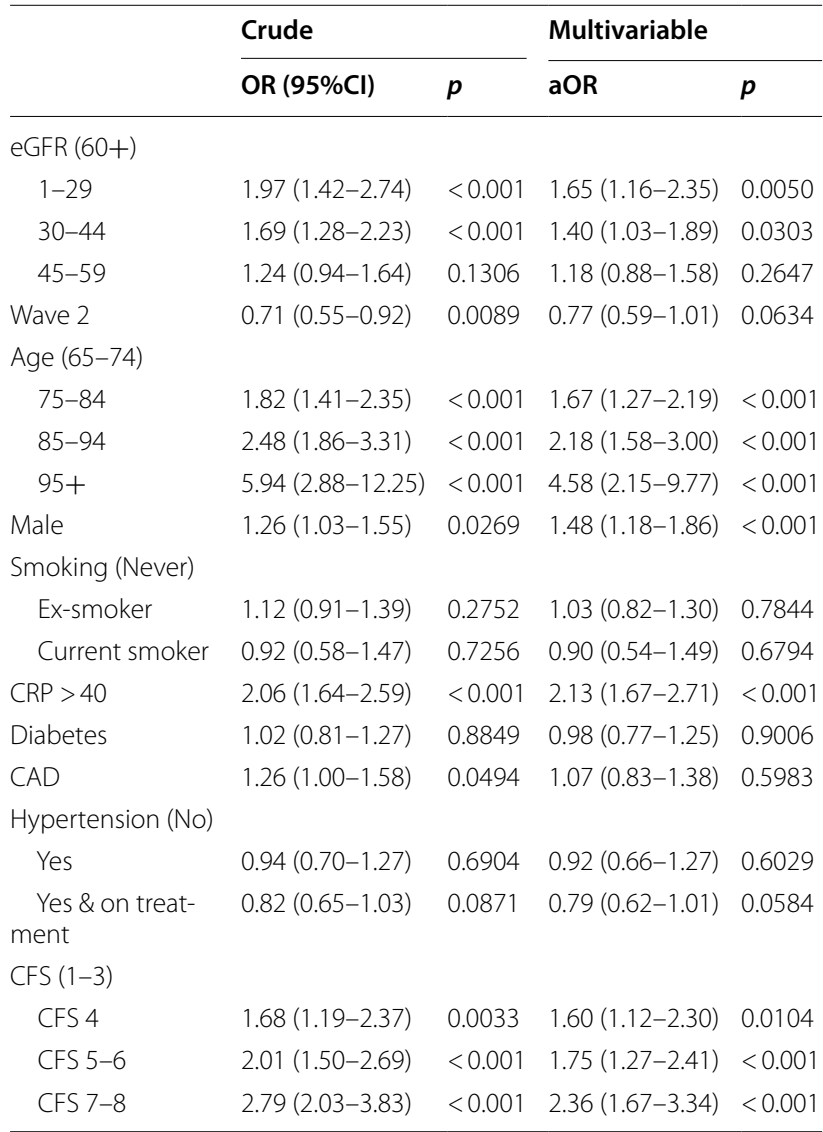

Note: aHR adjusted for eGFR, wave, age, sex, smoking status, CRP, diabetes, CAD, hypertension and CFS

pertinent that supportive measures are instituted at the earliest opportunity in at risk older patients to prevent further decline. We have identified eGFR may offer an improved prognostic indicator and at seemingly modest decline in renal function for this vulnerable patient cohort.

There are implications of our findings on future research. It is important to better understand the longer-term impact of COVID-19 in those with reduced renal function in survivors, and whether there are both immediate and longer term impacts on clinical outcomes in patients who survive. Further understanding of the impact of renal decline should also be assessed with other clinically important outcomes, such as quality of life, which requires further evaluation. Future research is needed into interventions to improve deranged renal function in older adults. 
Table 4 Crude and multivariable Cox proportional hazards regression, presenting the crude Hazard Ratio (HR) and adjusted $\mathrm{HR}(\mathrm{aHR})$ for the time to discharge

\begin{tabular}{|c|c|c|c|c|}
\hline & \multicolumn{2}{|l|}{ Crude } & \multicolumn{2}{|l|}{ Multivariable } \\
\hline & HR (95\%Cl) & $p$ & aHR & $p$ \\
\hline \multicolumn{5}{|l|}{ eGFR (60+) } \\
\hline $1-29$ & $0.79(0.61-1.03)$ & 0.0796 & $0.84(0.64-1.09)$ & 0.1833 \\
\hline $30-44$ & $0.97(0.79-1.20)$ & 0.8071 & $1.09(0.88-1.35)$ & 0.4255 \\
\hline $45-59$ & $0.99(0.82-1.20)$ & 0.9433 & $1.09(0.89-1.32)$ & 0.4121 \\
\hline Wave 2 & $0.61(0.51-0.73)$ & $<0.001$ & $0.60(0.50-0.73)$ & $<0.001$ \\
\hline \multicolumn{5}{|l|}{ Age (65-74) } \\
\hline $75-84$ & $0.85(0.72-0.99)$ & 0.0417 & $0.89(0.75-1.06)$ & 0.1830 \\
\hline $85-94$ & $0.70(0.58-0.85)$ & $<0.001$ & $0.72(0.58-0.89)$ & 0.0022 \\
\hline $95+$ & $0.64(0.33-1.25)$ & 0.1935 & $0.77(0.39-1.51)$ & 0.4405 \\
\hline Male & $0.97(0.84-1.12)$ & 0.7133 & $0.93(0.80-1.08)$ & 0.3394 \\
\hline \multicolumn{5}{|l|}{ Smoking (Never) } \\
\hline Ex-smoker & $1.07(0.93-1.24)$ & 0.3373 & $1.10(0.94-1.28)$ & 0.2235 \\
\hline Current smoker & $0.98(0.71-1.35)$ & 0.9104 & $0.97(0.70-1.35)$ & 0.8677 \\
\hline $\mathrm{CRP}>40$ & 1.15 (0.99-1.33) & 0.0712 & $1.07(0.92-1.24)$ & 0.4077 \\
\hline Diabetes & $0.91(0.78-1.06)$ & 0.2364 & $0.92(0.78-1.09)$ & 0.3529 \\
\hline CAD & $0.96(0.82-1.13)$ & 0.6435 & $1.01(0.85-1.20)$ & 0.9002 \\
\hline \multicolumn{5}{|l|}{ Hypertension (No) } \\
\hline Yes & $0.87(0.71-1.08)$ & 0.2059 & $0.97(0.78-1.20)$ & 0.7580 \\
\hline Yes \& on treatment & $1.09(0.93-1.28)$ & 0.2812 & $1.08(0.92-1.28)$ & 0.3466 \\
\hline \multicolumn{5}{|l|}{ CFS (1-3) } \\
\hline CFS 4 & $0.83(0.66-1.03)$ & 0.0960 & $0.89(0.71-1.12)$ & 0.3103 \\
\hline CFS 5-6 & $0.71(0.59-0.85)$ & $<0.001$ & $0.78(0.64-0.95)$ & 0.0157 \\
\hline CFS 7-8 & $0.71(0.57-0.87)$ & 0.0014 & $0.79(0.63-0.99)$ & 0.0380 \\
\hline
\end{tabular}

Note: aHR adjusted for eGFR, wave, age, sex, smoking status, CRP, diabetes, CAD, hypertension and CFS

\section{Conclusion}

Point of care renal failure during admission to hospital, measured by eGFR is a helpful independent predictor of mortality in older patients admitted to hospital with COVID-19. Importance should be placed on either a suspected dose-response, or the clinical implications of increased management may be triggered by Stage $3 \mathrm{~b}$ renal failure.

\section{Abbreviations}

aHR: Adjusted hazard ratio; aOR: Adjusted odds ratio; AKI: Acute kidney injury; CFS: Clinical frailty scale; Cl: 95\% confidence interval; CRP: C-reactive protein; CKD: Chronic kidney disease; CKD-EPI: Chronic Kidney Disease, Creatinine Equation; COPE: COVID-19 in older people study; eGFR: Estimated glomerular filtration rate; HR: Hazard ratio; HRA: Health research authority; IQR: Interquartile ranger; OR: Odds ratio.

\section{Supplementary Information}

The online version contains supplementary material available at https://doi. org/10.1186/s12877-022-02782-5.

\section{Additional file 1.}

\section{Acknowledgements}

COPE Team Members (collaborators)

Aberdeen Royal Infirmary: Eilidh Bruce, Alice Einarsson, Kirsty McCrorie.

Glasgow Royal Infirmary: Ken Aggrey, Jimmy Bilan, Kerr Hartrop, Caitlin Murphy, Aine McGovern.

Hospital Policlinico Modena, University of Modena and Reggio Emilia, Italy: Prof Enrico Clini, Prof Giovanni Guaraldi, Alessia Verduri. Inverclyde Royal Infirmary Carly Bisset, Ross Alexander. King's College London: Joanna Kelly, Caroline Murphy.

North Bristol NHS Trust: Tarik El Jichi Mutasem, Sandeep Singh, Dolcie Paxton, Will Harris.

Royal Alexandra Hospital (Paisley): Prof Susan Moug, Norman Galbraith, Emma Bhatti, Jenny Edwards, Siobhan Duffy.

Salford Royal Hospital: Maria Fernanda Ramon Espinoza, Thomas Kneen, Anna Dafnis, Hala Allafi, Maria Narro Vidal, Angeline Price, Lyndsay Pearce, Thomas Lee, Shefali Sangani, Madeline Garcia.

Ysbyty Tystad Fawr: Charlotte Davey, Sheila Jones, Kiah Lunstone, Alice Cavenagh, Charlotte Silver, Thomas Telford, Rebecca Simmons.

\section{Authors' contributions}

$\mathrm{BC}$ conceived the study. JH and $\mathrm{KM}$ are CO-Cls of COPE. The protocol was drafted by $A V M, B C, J H, K H, P B, P K M$, and TQ, RS and analysed the data. $B C$ is the statistical lead and PKM is the steering committee chair of COPE studies. AK, EAR, JL, SG did literature review and wrote the first draft under the supervision of BC and PKM. All authors contributed in drafting, reviewing and approved the final version of the manuscript for submission. $\mathrm{JH}$ is the guarantor.

\section{Funding}

No funding declared for this study. The Study was sponsored by Cardiff University who had no role on the study design, analysis or interpretation of the findings. BC is partially supported by the NIHR Maudsley Biomedical Research Centre at South London Maudsley NHS Foundation Trust, and King's College London. EAR received the Gwyn Seymour Scholarship from the Department of Medicine for the Elderly, NHS Grampian, as part of the Aberdeen Summer Research Scholarship Scheme, Aberdeen Clinical Academic Training Programme, University of Aberdeen.

\section{Availability of data and materials}

Due to the confidential nature of the data used within this study, the data is not available for secondary data analysis.

\section{Declarations}

\section{Ethics approval and consent to participate}

Approval for the study was granted in the UK by the Health Research Authority (HRA) (20/HRA/1898) to Cardiff University and in Italy from the Ethics Committee of Hospital Policlinico Modena (Reference 369/2020/OSS/ AOUMO). HRA was granted to access patient patient records, and no Patient level consent was not required since this study was carried by patient note and hospital record review.

\section{Consent for publication}

Not applicable.

\section{Competing interests}

EAR received the Gwyn Seymour Scholarship from the Department of Medicine for the Elderly, NHS Grampian, as part of the Aberdeen Summer Research Scholarship Scheme, Aberdeen Clinical Academic Training Programme, University of Aberdeen. The remaining authors declare no competing interests.

\section{Author details}

${ }^{1}$ Biostatistics and Health Informatics, Institute of Psychiatry, Psychology \& Neuroscience, King's College London, De Crespigny Park, London SE5 8AF, UK. ${ }^{2}$ Ageing Clinical \& Experimental Research Team, Institute of Applied Health Sciences, University of Aberdeen, Aberdeen, Scotland AB25 2ZD. ${ }^{3}$ Forensic and Neurodevelopmental Sciences, Institute of Psychiatry, Psychology \& Neuroscience, King's College London, De Crespigny Park, London SE5 8AF, UK. ${ }^{4}$ Department of Geriatric Medicine; Ysbyty Ystrad Fawr, Aneurin Bevan University Health Board, Hengoed, Wales CF82 7GP. ${ }^{5}$ Department of Geriatric 
Medicine, Glasgow Royal Infirmary, NHS Greater Glasgow \& Clyde, G4 OSF, Glasgow, UK. ${ }^{6}$ Ageing and Complex Medicine Department, Salford Royal NHS Foundation Trust, Stott Lane, Manchester M6HD8, UK. ${ }^{7}$ Department of Surgery and Care of the Elderly, Southmead Hospital, North Bristol NHS Trust, Bristol BS10 5NB, UK. ${ }^{8}$ Institute of Cardiovascular and Medical Sciences, University of Glasgow, Glasgow G12 8TA, UK. ${ }^{9}$ Division of Population Medicine, Cardiff University, Cardiff Cf10 3XQ, UK.

Received: 27 September 2021 Accepted: 24 January 2022

Published online: 12 February 2022

\section{References}

1. Wang D, Hu B, Hu C, et al. Clinical Characteristics of 138 Hospitalized Patients With 2019 Novel Coronavirus-Infected Pneumonia in Wuhan, China. JAMA. 2020;323(11):1061.

2. Wang L, Li X, Chen H, et al. Coronavirus disease 19 infection does not result in acute kidney injury: an analysis of 116 hospitalized patients from Wuhan, China. Am J Nephrol. 2020;51(5):343-8.

3. Pei G, Zhang Z, Peng J, et al. Renal involvement and early prognosis in patients with COVID-19 pneumonia. J Am Soc Nephrol. 2020;31(6):1157-65

4. Su H, Yang M, Wan C, et al. Renal histopathological analysis of 26 post-mortem findings of patients with COVID-19 in China. Kidney Int. 2020;98(1):219-27.

5. Nadim M, Forni L, Mehta R, et al. COVID-19-associated acute kidney injury: consensus report of the 25th acute disease quality initiative (ADQI) workgroup. Nat Rev Nephrol. 2020;16(12):747-64.

6. Levey A, Stevens $L$, Schmid C, et al. A new equation to estimate glomerular filtration rate. Ann Intern Med. 2009;150(9):604

7. Chertow G, Burdick E, Honour M, Bonventre J, Bates D. Acute kidney injury, mortality, length of stay, and costs in hospitalized patients. J Am Soc Nephrol. 2005;16(11):3365-70.

8. Hamilton P, Hanumapura P, Castelino L, et al. Characteristics and outcomes of hospitalised patients with acute kidney injury and COVID-19. PLoS One. 2020;15(11):e0241544.

9. Paek J, Kim Y, Park W, et al. Severe acute kidney injury in COVID19 patients is associated with in-hospital mortality. PLoS One. 2020:15(12):e0243528

10. Trabulus S, Karaca C, Balkan I, et al. Kidney function on admission predicts in-hospital mortality in COVID-19. PLoS One. 2020;15(9):e0238680.

11. Xiang H, Fei J, Xiang $Y$, et al. Renal dysfunction and prognosis of COVID-19 patients: a hospital-based retrospective cohort study. BMC Infect Dis. 2021;21(1):158. https://doi.org/10.1186/s12879-021-05861.

12. Uribarri A, Núñez-Gil I, Aparisi A, et al. Impact of renal function on admission in COVID-19 patients: an analysis of the international HOPE COVID-19 (health outcome predictive evaluation for COVID 19) registry. J Nephrol. 2020;33(4):737-45

13. Age and frailty are independently associated with increased COVID-19 mortality and increased care needs in survivors: results of an international multi-Centre study. Geriatric medicine research collaborative; Covid collaborative; Welch C. Age Ageing. 2021;50(3):617-30.

14. Becerra-Muñoz V, Núñez-Gil I, Eid C, et al. Clinical profile and predictors of in-hospital mortality among older patients hospitalised for COVID-19. Age Ageing. 2020;50(2):326-34.

15. Zerah L, Baudouin É, Pépin M, et al. Clinical characteristics and outcomes of 821 older patients with SARS-Cov-2 infection admitted to acute care geriatric wards. J Gerontol A Biol Sci Med Sci. 2020;76(3):e4-e12.

16. Hewitt J, Carter B, Vilches-Moraga A, et al. The effect of frailty on survival in patients with COVID-19 (COPE): a multicentre, European, observational cohort study. Lancet Public Health. 2020;5(8):e444-51.

17. Zhou F, Yu T, Du R, et al. Clinical course and risk factors for mortality of adult inpatients with COVID-19 in Wuhan, China: a retrospective cohort study. Lancet. 2020;395(10229):1054-62.

18. Hirsch J, Ng J, Ross D, et al. Acute kidney injury in patients hospitalized with COVID-19. Kidney Int. 2020;98(1):209-18.

19. Lim J, Park S, Jeon Y, et al. Fatal outcomes of COVID-19 in patients with severe acute kidney injury. J Clin Med. 2020;9(6):1718.

20. Cheng Y, Luo R, Wang $K$, et al. Kidney disease is associated with inhospital death of patients with COVID-19. Kidney Int. 2020;97(5):829-38.
21. Xu H, Garcia-Ptacek S, Annetorp M, et al. Acute kidney injury and mortality risk in older adults with COVID-19. J Nephrol. 2021;34(2):295-04.

22. Price A, Barlow-Pay F, Duffy S, et al. Study protocol for the COPE study: COVID-19 in older PEople: the influence of frailty and multimorbidity on survival. A multicentre, European observational study. BMJ Open. 2020;10(9):e040569.

23. Carter B, Collins JT, Barlow-Pay F, et al. Nosocomial COVID-19 infection: examining the risk of mortality. The COPE-nosocomial study (COVID in older PEople). COPE study collaborators. J Hosp Infect. 2020;106(2):376-84

24. Stevens PE, Levin A. KDIGO Guideline development work group members. Evaluation and management of chronic kidney disease: synopsis of the kidney disease: improving global outcomes 2012 clinical practice guideline. Ann Intern Med. 2013;158(11):825-30.

25. Stringer $D$, Braude $P$, Myint $P$, et al. The role of $C$-reactive protein as a prognostic marker in COVID-19. Int J Epidemiol. 2021;50(2):420-9.

26. Rockwood K. A global clinical measure of fitness and frailty in elderly people. CMAJ. 2005;173(5):489-95.

27. Rockwood K, Theou O. Using the clinical frailty scale in allocating scarce health care resources. Can Geriatr J. 2020:23(3):254-9.

28. Williamson E, Walker A, Bhaskaran K, et al. Factors associated with COVID19-related death using OpenSAFELY. Nature. 2020;584(7821):430-6.

29. Clinical guide for acute kidney injury in hospitalised patients with COVID19 outside the intensive care unit during the coronavirus pandemic. NHS England and NHS Improvement 2020 (online). Available at: https://www. nice.org.uk/ Accessed 20 July, 2021.

30. Cohen J, South A, Shaltout H, Sinclair M, Sparks M. Renin-angiotensin system blockade in the COVID-19 pandemic. Clin Kidney J. 2021;14(Supplement_1):i48-59.

31. Channappanavar R, Perlman S. Pathogenic human coronavirus infections: causes and consequences of cytokine storm and immunopathology. Semin Immunopathol. 2017;39(5):529-39.

32. Fu Y, Cheng Y, Wu Y. Understanding SARS-CoV-2-mediated inflammatory responses: from mechanisms to potential therapeutic tools. Virol Sin. 2020;35(3):266-71.

33. Statement on the eighth meeting of the International Health regulations (2005) Emergency Committee regarding the coronavirus disease (COVID19) pandemic 15 July 2020. Available at: https://www.who.int Accessed 25 July 2021

34. Glassock R, Rule A. The implications of anatomical and functional changes of the aging kidney: with an emphasis on the glomeruli. Kidney Int. 2012:82(3):270-7.

35. Betjes M. Immune cell dysfunction and inflammation in end-stage renal disease. Nat Rev Nephrol. 2013:9(5):255-65.

\section{Publisher's Note}

Springer Nature remains neutral with regard to jurisdictional claims in published maps and institutional affiliations.

Ready to submit your research? Choose BMC and benefit from:

- fast, convenient online submission

- thorough peer review by experienced researchers in your field

- rapid publication on acceptance

- support for research data, including large and complex data types

- gold Open Access which fosters wider collaboration and increased citations

- maximum visibility for your research: over 100M website views per year

At BMC, research is always in progress.

Learn more biomedcentral.com/submissions 